\title{
Detumbling Large Space Debris via Laser Ablation
}

\author{
Massimo Vetrisano, Nicolas Thiry and Massimiliano Vasile \\ Space ART \\ Advanced Space Concepts Laboratory \\ Department of Mechanical \& Aerospace Engineering \\ University of Strathclyde \\ James Weir Building, 75 Montrose Street, University of Strathclyde| Glasgow | G1 1XJ | \\ \{massimo.vetrisano, nicolas.thiry, massimiliano.vasile\}@strath.ac.uk
}

\begin{abstract}
This paper presents an approach to control the rotational motion of large space debris (the target) before the spacecraft starts deflecting its trajectory through laser ablation. A rotational control strategy based on the instantaneous angular velocity of the target is presented. The aim is to impart the maximum control torque in the direction of the instantaneous angular velocity while minimizing the undesired control components in the other directions. An on-board state estimation and control algorithm is then implemented. It simultaneously provides an optimal control of the rotational motion of the target through the combination of a LIDAR and a navigation camera. The instantaneous angular velocity of the debris is estimated through the application of the optic flow technique. The whole control and estimation technique is applied to the case of cylindrical and parallelepiped shapes as representative of upper stages and spacecrafts. When applied to the cylindrical shape, results show that the control strategy and laser technique fail to control along three directions unless the geometrical axes are different from the inertial ones. In general the thrust vector is aligned with the normal to the local surface meaning that no control torque can be exerted along the longitudinal axis in the case of an ideal cylinder.
\end{abstract}

\section{TABLE OF CONTENTS}

1. INTRODUCTION .1

2. LASER MODEL 1

3. ROTATIONAL MOTION CONTROL .......................3 4. DEBRis Rotational MOTION

RECONSTRUCTION....................................................3 5. RESULTS..................................................................5

BIOGRAPHY ..........................................................

\section{Introduction}

From the very beginning of space flight in 1957, a troubling legacy of garbage has been left in space. Discarded rocket bodies, derelict satellites, and debris from explosions or collisions now litter Earth orbits. Space debris pose a risk to human and robotic space flight, commercial space activities, and even safety of humans on the ground. In recent years it has become clear that the increasing population of space debris could lead to catastrophic consequences in the near term. The Kessler syndrome (where the density of objects in orbit is high enough that collisions could set off a cascade)

978-1-4799-5380-6/15/\$31.00 @2015 Crown is more realistic than when it was first proposed in 1978. A collision between rockets' upper stages and large spacecraft (e.g. Envisat-4) could potentially produce huge clouds of debris in Low Earth and Highly Elliptical Orbits (LEO and HEO).

It is paramount that collisions between such objects have to be avoided actively in order to safeguard the access to LEO and HEO and the safety of spacecraft already orbiting there. A set of techniques have been proposed to deflect and deorbit existing space debris, spanning from attaching a device as in [1] or using contactless techniques, such using the thruster plume impingement. Independently from the chosen technique, a common problem is represented by the fact that big space items could be tumbling at a relatively high rate at the time the deflection is imparted.

In this paper we tackle this detumbling problem by using a laser ablation method, considering the former study of [2], where a laser was used to deflect an asteroid while decreasing its angular velocity. In fact, as it will be shown in Section 2, it is necessary to reduce the angular rate because of the effectiveness of this method is affected by the local surface velocity. The proposed rotational control law is based on the instantaneous angular velocity as described Section 3. For this reason an optical flow method to estimate this quantity was implemented in Section 4. Given the fact that the laser ablation process is also affected by the shape of the debris, Section 5 will show how the size and the shape have an impact on the detumbling time. In particular a parallelepiped and a cylinder will be considered, using available data from the tumbling motion of the Envisat-4 spacecraft. Finally Section 7 provides a summary and a plan for future works.

\section{Laser Model}

This section outlines the ablation model used to predict the effect of the ablation process on the spacecraft. For further details, the interested reader can find an exhaustive description in [3], [4] and [5].

The force due to the laser $F_{L}$ is given by the product of the velocity of the ejected gas $\bar{v}$ and the mass flow rate of the ablated material $\dot{m}$ :

$$
F_{L}=\lambda \bar{v} \dot{m}
$$

where $\lambda=0.88$ is a constant scattering factor used to account for the non-unidirectional expansion of the ejecta. The mass flow rate is given by the integral, over the area illuminated by the laser, of the mass flow rate per unit area $\dot{\mu}:$ 


$$
\dot{m}=2 V_{\text {rot }} \int_{0}^{y_{\max }} \int_{t_{\text {in }}}^{t_{\text {out }}} \dot{\mu} d t d y
$$

where $V_{\text {rot }}$ is the speed at which the surface of the debris is moving under the spotlight, $y_{\max }$ is the maximum width of the spot and $t_{\text {in }}$ and $t_{\text {out }}$ are the entry and existence times from the spotlight of a point of the surface moving with velocity $V_{\text {rot }}$. The mass flow rate $\dot{\mu}$ per unit area is expressed as:

$$
E_{v}^{*} \dot{\mu}=P_{I N}-Q_{R A D}-Q_{C O N D}
$$

where $P_{I N}$ is the absorbed laser power density, $E_{v}^{*}$ is an augmented vaporization enthalpy, $Q_{C O N D}$ the conduction and $Q_{R A D}$ the radiation fluxes. The augmented enthalpy $E_{v}^{*}=E_{v}^{*}\left(T_{0}, T_{s}, C_{p}, C_{v}, \bar{v}\right)$ depends on the initial surface temperature $T_{0}$, the temperature $T_{S}$ of the ablation front, the heat capacity of solid phase $C_{v}$, vapour phase $C_{p}$ and the mean ejection velocity $\bar{v}$.

The input power $P_{I N}$ in Eq. (3) is computed assuming that the beam is generated by an electrically pumped laser. The electric power is generated by a solar array with conversion efficiency $\eta_{s}$. The electric power is then converted into laser power with efficiency $\eta_{L}$. The surface of the spacecraft is absorbing only the fraction $\alpha_{M}=\left(1-\alpha_{s}\right)$ of the incoming light, where $\alpha_{s}$ is the albedo at the frequency of the laser light. One can consider this as the worst case scenario. The absorbed power per square meter at the spot is therefore:

$$
P_{I N}=\tau \tau_{g} \alpha_{M} \eta_{P} \eta_{L} \eta_{S} \frac{P_{1 A U} A_{S A}}{A_{S p o t} r_{A U}^{2}}
$$

where $\tau$ is a degradation factor due to contamination, $\eta_{P}$ is the efficiency of the power system, $P_{1 A U}$ is the solar constant at 1 Astronomical Unit (AU), $A_{\mathrm{SA}}$ is the area of the solar arrays, $A_{\text {spot }}$ is the area of the spot and $r_{A U}$ is the distance from the Sun measured in AU. The term $\tau_{g}$ accounts for the fraction of laser light absorbed by the ejected gas. The degradation factor can be computed by following [6] and taking the plume density $\rho_{\text {plume }}(r, \xi)$ at any given distance $r$ from the spot location, and elevation angle $\xi$ from the surface normal. The ejecta thickness on any exposed surface, $h$, grows linearly with the mean ejection velocity at surface of the spacecraft $\bar{v}$ and the density of the plume $\rho_{\text {plume }}(r, \xi)$, which decreases as the distance from the spacecraft increases [5]. The increasing thickness of the contaminants will ultimately reduce the power generated by the solar arrays and, therefore, the laser output power. The consequence is a reduction of the thrust imparted onto the spacecraft until the ablation process ceases completely and the thrust with it. The reduction of the power generated by the solar arrays, $\tau$, can be computed from the Beer-Lambert-Bougier law:

$$
\tau=e^{-\eta h}
$$

where $\eta$ is the absorbance per unit length of the accumulated ejecta. Nonetheless the expected impingement for short operations time for an object as debris can be assumed almost negligible.

Table 1 reports the parameters used for the calculation of Eq.(4) and (5).

Table 1. Laser system coefficients.

\begin{tabular}{|c|c|}
\hline Parameter & Value \\
\hline$\tau_{g}$ & 1 \\
\hline$\alpha_{M}$ & 0.84 \\
\hline$\eta_{P}$ & 0.85 \\
\hline$\eta_{L}$ & 0.55 \\
\hline$\eta_{S}$ & 0.3 \\
\hline$\eta$ & $2 \cdot 10^{-4} \mathrm{~cm}^{-1}$ \\
\hline
\end{tabular}

The distance from the focal point, along the beam, at which the beam radius is $w_{0} \sqrt{2}$ (known as Rayleigh length, [7]), with $w_{0}$ the radius at the focal point, is about $3 \mathrm{~m}$, assuming a $50 \mathrm{~mm}$ in diameter focusing mirror.

If one assumes a constant input power of $860 \mathrm{~W}$, a nominal spot size of $0.8 \mathrm{~mm}$ [5], an optics designed to focus the beam at a nominal distance of $50 \mathrm{~m}$ from the laser source and $3 \mathrm{~m}$ mean size of the object, then the thrust due to the sublimation process with respect to the laser source distance from the spot and $V_{r o t}$ has the trend represented in Figure 1. As for the simulations, we considered that the ablated material is aluminium-lithium alloy, whose mean density is $2.150 \mathrm{~kg} / \mathrm{m}^{3}$

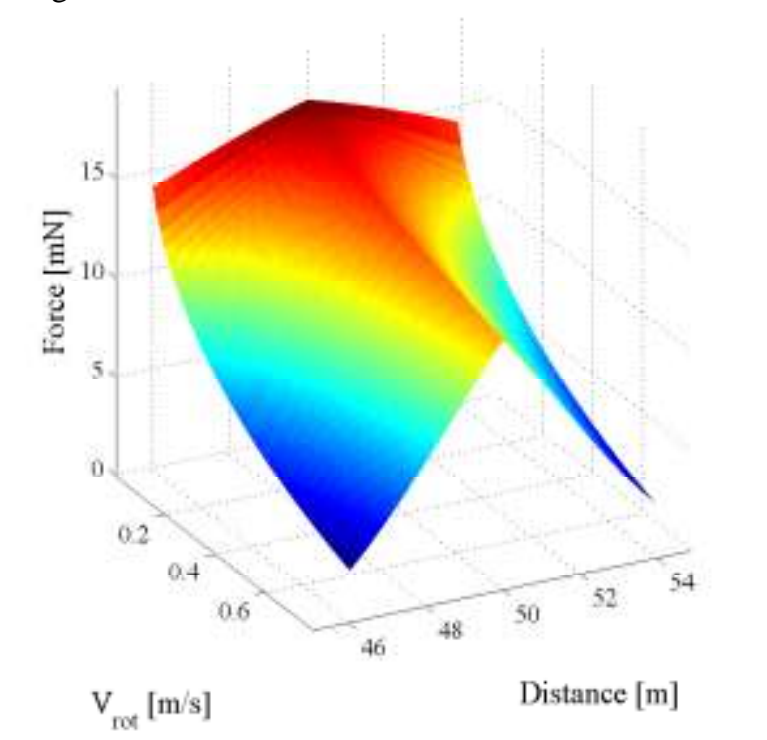

Figure 1. Force due to the sublimation process with respect 
to the laser distance from the spot and the velocity of the surface under the spot light.

As one can see, the force increases as the tangential velocity decreases and an absolute maximum is reached when $V_{\text {rot }}$ is zero and the distance equals the focusing length. Moreover, for higher values of velocity, moving by 3-4 $\mathrm{m}$ with respect to the focusing length causes a reduction of about $70 \%$ of the nominal value. This case will occur at the beginning of the operations while, as the angular velocity decreases, the variation of the force with the distance will be less pronounced.

\section{Rotational Motion Control}

We considered the pure rotational dynamics of the tumbling object. Its rotational motion is governed by the following system of differential equations:

$$
\begin{aligned}
& \dot{\mathbf{q}}=\frac{1}{2} \Pi \mathbf{q} \\
& \mathbf{I} \dot{\boldsymbol{\omega}}+\boldsymbol{\omega} \times \mathbf{I} \boldsymbol{\omega}=\mathrm{M}_{c}
\end{aligned}
$$

where $\mathbf{q}=\left[\begin{array}{llll}q_{1} & q_{2} & q_{3} & q_{4}\end{array}\right]^{T}$ is the quaternions vector, $\boldsymbol{\omega}=\left[\begin{array}{lll}\omega_{x} & \omega_{y} & \omega_{z}\end{array}\right]^{T}$ is the angular velocity vector in the body frame, $\mathrm{I}$ is the matrix of inertia of the spacecraft, $\mathbf{M}_{c}$ is the control torque, and $\Pi$ is given by:

$$
\boldsymbol{\Pi}=\left[\begin{array}{cccc}
0 & \omega_{z} & -\omega_{y} & \omega_{x} \\
-\omega_{z} & 0 & \omega_{x} & \omega_{y} \\
\omega_{y} & -\omega_{x} & 0 & \omega_{z} \\
-\omega_{1} & -\omega_{y} & -\omega_{z} & 0
\end{array}\right]
$$

Perturbative torques from the Sun light pressure, magnetic field and gravity gradient was not taken into account (because their cumulative effect is negligible with respect to the torque induced by the laser).

With reference to Figure 2, a strategy to reduce the spinning rate, already proved to work for the case of an asteroid [2] is to apply a control torque proportional to the opposite of the angular velocity vector:

$$
\mathbf{M}_{c} \propto \frac{\omega}{\|\omega\|}
$$

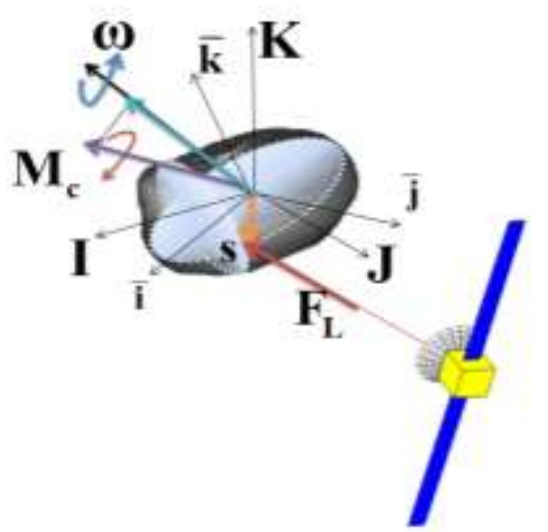

Figure 2. Angular velocity control scheme for space debris via laser ablation

The actual control torque $\mathbf{M}_{c}$ that can be generated is given by the cross product of the thrust $\mathbf{F}_{L}^{b}$ with the position vector $s$ :

$$
\mathbf{M}_{c}=\mathbf{s} \times \mathbf{F}_{L}^{b}=\left\{\begin{array}{l}
M_{c-x}^{b}=-F_{z}^{b} s_{y}^{b}+F_{y}^{b} s_{z}^{b} \\
M_{c-y}^{b}=F_{z}^{b} s_{x}^{b}-F_{x}^{b} s_{z}^{b} \\
M_{c-z}^{b}=-F_{y}^{b} s_{x}^{b}+F_{x}^{b} s_{y}^{b}
\end{array}\right.
$$

where thrust $\mathbf{F}_{L}^{b}$ is the thrust vector, projected in body axes, produced by the ablation process at point $s$ on the surface of the object. We know that the force exerted by the laser is perpendicular to the local normal. In this way knowing the shape of the object and, thus, the direction of the normals to its surface is sufficient for determining the direction of all the possible control torques. Following the results of [2], we point the laser with the following control logic

$$
\min _{s} \mathbf{M}_{\boldsymbol{c}} \overline{(\boldsymbol{s})} \cdot \boldsymbol{\omega}
$$

This means that the laser will hit the points on the surface of the object, where the misalignment of the control torque with respect to the angular velocity will be the minimum.

\section{Debris Rotational Motion Reconstruction}

In order to control the rotational motion of the debris, it is necessary to estimate its instantaneous angular velocity. Tracking feature points on the asteroid's surface can be used to measure the asteroid's angular velocity.

By applying the time derivative to both sides of the pin-hole camera model in Eq.(6), one can relate the optical flow with the angular and linear velocity of the asteroid: 


$$
\left[\begin{array}{c}
\dot{u} \\
\dot{v}
\end{array}\right]=\frac{f}{x_{c}}\left[\begin{array}{c}
\frac{d y_{c}}{d t} \\
\frac{d z_{c}}{d t}
\end{array}\right]-\frac{f}{x_{c}^{2}} \frac{d x_{c}}{d t}\left[\begin{array}{l}
y_{c} \\
z_{c}
\end{array}\right]
$$

with:

$$
\left[\begin{array}{lll}
\frac{d x_{c}}{d t} & \frac{d y_{c}}{d t} & \frac{d z_{c}}{d t}
\end{array}\right]=-\boldsymbol{\omega}_{B / C} \times \mathbf{p}_{c}^{a}-\mathbf{V}_{B / C}
$$

The vectors $\mathbf{V}_{B / C}$ and $\boldsymbol{\omega}_{B / C}$ are respectively the linear and angular velocities of the asteroid relative to the camera, assuming that the camera is static in Hill's reference frame. The relative velocity vector is defined as:

$$
\mathbf{V}_{B / C}=\mathbf{R}_{H C} \delta \dot{\mathbf{r}}^{h}
$$

The vector $\mathbf{p}_{c}^{a}=\left[x_{c}^{a}, y_{c}^{a}, z_{c}^{a}\right]^{T}$ gives the position of a point on the surface of the asteroid with respect to the centre of Hill's reference frame, projected onto the reference frame of the camera:

$$
\mathbf{p}_{c}^{a}=\mathbf{R}_{H C} \mathbf{x}_{\text {surface }}
$$

For a single point on the surface of the asteroid Eq. (6) can now be written as:

$$
\left[\begin{array}{c}
\dot{u} \\
\dot{v}
\end{array}\right]=\mathbf{M}\left(f, \mathbf{r}_{c}, \mathbf{p}_{c}^{a}\right)\left[\begin{array}{c}
\mathbf{V}_{B / C} \\
\boldsymbol{\omega}_{B / C}
\end{array}\right]
$$

with:

$$
\mathbf{M}\left(f, \mathbf{r}_{c}, \mathbf{p}_{c}^{a}\right)=\left[\begin{array}{cccccc}
\frac{u}{x_{c}} & -\frac{f}{x_{c}} & 0 & -v & -\frac{u v}{f} & \frac{u^{2}}{f}+f \frac{x_{c}^{a}}{x_{c}} \\
\frac{v}{x_{c}} & 0 & -\frac{f}{x_{c}} & u & -\frac{v^{2}}{f}-f \frac{x_{c}^{a}}{x_{c}} & \frac{u v}{f}
\end{array}\right]
$$

The angular velocity can be obtained directly from Eq. (61) or by re-arranging the equation so that the angular velocity becomes a function of the linear velocity:

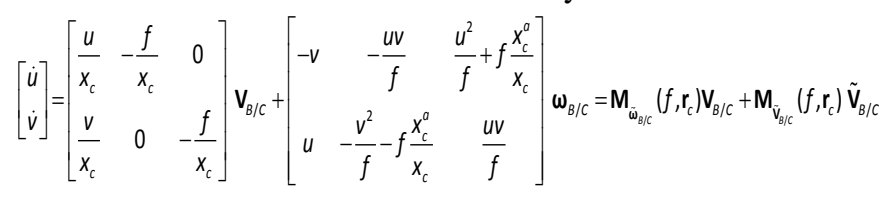

$\mathbf{M}_{\tilde{\omega}_{B / C}}\left(f, \mathbf{r}_{c}\right)$ and $\mathbf{M}_{\tilde{v}_{B / C}}\left(f, \mathbf{r}_{c}\right)$ are the partitions of $\mathbf{M}\left(f, \mathbf{r}_{c}\right)$

relatively to the angular and linear velocity of the asteroid. The algorithm requires the knowledge of the relative position and relative attitude between the spacecraft and the asteroid to determine the relative position of each feature points. The relative position and velocity of the spacecraft with respect to the centre of mass of the asteroid can be extracted through a proximity motion reconstruction, which we modelled in terms of a perturbed state vector.

It is here assumed that the centre of mass of the debris is known with an error $\mathbf{x}_{C o M}^{\text {bias }}$. Furthermore, it is assumed that the attitude of the asteroid at time $t_{0}$ and the position of each surface point with respect to the centre of mass are obtained from an observation campaign prior to the beginning of the ablation process or from available CAD models of the tumbling object. An estimation of the $\mathbf{p}_{c}^{a}$ vector at any time during the ablation process can be obtained from:

$\tilde{\mathbf{p}}_{c}^{a}=\left[\begin{array}{ccc}\tilde{x}_{c}^{a} & \tilde{y}_{c}^{a} & \tilde{z}_{c}^{a}\end{array}\right]=\mathbf{R}_{S C-\text { attitude }} \mathbf{R}_{H C} \tilde{\boldsymbol{x}}_{\text {suffoce }}=\mathbf{R}_{S C-\text { attitude }} \mathbf{R}_{H C} \mathbf{R}\left(\tilde{\mathbf{q}}, \tilde{\mathbf{q}}_{0}\right)\left(\mathbf{x}_{\text {suffoce }}^{0}+\mathbf{x}_{\text {com }}^{\text {bias }}+\boldsymbol{\zeta}_{\text {suffoce }}\right)$

where $\mathbf{x}_{\text {surface }}^{0}$ is the actual position of a feature point at $t_{0}$, $\boldsymbol{\zeta}_{\text {surface }}$ is an error which derives from the camera and LRF measurements required to build a three dimensional map of the asteroid, $\quad \mathbf{R}_{s c \text {-attitude }}$ is the attitude matrix of the spacecraft, which affects the pointing of the camera on two axes and $\mathbf{R}\left(\tilde{\mathbf{q}}_{k}, \tilde{\mathbf{q}}_{0}\right)$ is the rotation matrix from the initial asteroid's attitude $\tilde{\mathbf{q}}_{0}$ to the current attitude $\tilde{\mathbf{q}}$. The position of a feature point with respect to the camera then becomes:

$$
\begin{aligned}
\tilde{\mathbf{r}}_{c}=\left[\begin{array}{lll}
\tilde{x}_{c} & \tilde{y}_{c} & \tilde{z}_{c}
\end{array}\right] & =\mathbf{R}_{S C-\text { attitude }} \mathbf{R}_{H C} \mathbf{x}_{\text {Surf-SC}}=\mathbf{R}_{S C-\text { attitude }} \mathbf{R}_{H C}\left(\tilde{\mathbf{x}}_{\text {surface }}-\delta \tilde{\mathbf{r}}^{h}\right)= \\
& =\mathbf{R}_{S C \text {-attitude }} \mathbf{R}_{H C}\left(\mathbf{R}\left(\tilde{\mathbf{q}}_{k}, \tilde{\mathbf{q}}_{0}\right)\left(\mathbf{x}_{\text {surface }}^{0}+\mathbf{x}_{\text {CoM }}^{\text {bias }}+\boldsymbol{\varphi}_{\text {surface }}\right)-\delta \tilde{\mathbf{r}}^{h}\right)
\end{aligned}
$$

If one then introduces the pixelisation error then the two matrices in Eq.(12) become:

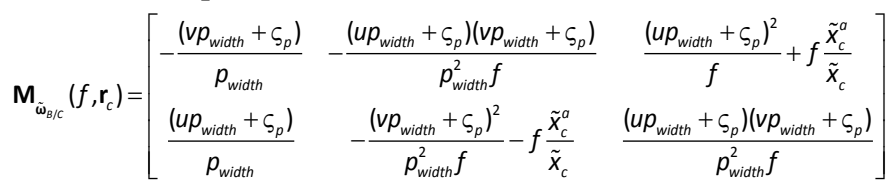

$$
\begin{aligned}
& \mathbf{M}_{\tilde{v}_{s e c}}\left(f, \mathbf{r}_{c}\right)=\left[\begin{array}{lll}
\frac{\left(u p_{\text {width }}+S_{p}\right)}{p_{\text {width }} \tilde{x}_{c}} & -\frac{f}{\tilde{x}_{c}} & 0 \\
\frac{\left(v p_{\text {width }}+S_{p}\right)}{p_{\text {width }} \tilde{x}_{c}} & 0 & -\frac{f}{\tilde{x}_{c}}
\end{array}\right]
\end{aligned}
$$

and $\dot{u}$ and $\dot{v}$ are approximated with $\Delta \mathbf{u} / \Delta t$ at two consecutive instants of time $k-1$ and $k$. The flow field then becomes:

$$
\left[\begin{array}{c}
\dot{u} \\
\dot{v}
\end{array}\right]=\left[\begin{array}{l}
\left(u^{k} p_{\text {width }}+\varsigma_{p}^{k}\right)-\left(u^{k-1} p_{\text {width }}+\varsigma_{p}^{k-1}\right) \\
\left(v^{k} p_{\text {width }}+\varsigma_{p}^{k}\right)-\left(v^{k-1} p_{\text {width }}+\varsigma_{p}^{k-1}\right)
\end{array}\right] /\left(p_{\text {width }} \Delta t\right)
$$

Introducing Eqs.(13), (14), (15) and (16) into Eq. (12) and solving for the angular velocity give: 


$$
\begin{aligned}
\tilde{\boldsymbol{\omega}}_{B / C} & =\left[\begin{array}{ccc}
-v & -\frac{u v}{f} & \frac{u^{2}}{f}+f \frac{\tilde{x}_{c}^{a}}{\tilde{x}_{c}} \\
u & -\frac{v^{2}}{f}-f \frac{\tilde{x}_{c}^{a}}{\tilde{x}_{c}} & \frac{u v}{f}
\end{array}\right]^{0}\left(\left[\begin{array}{l}
\dot{u} \\
\dot{v}
\end{array}\right]-\left[\begin{array}{ccc}
\frac{u}{\tilde{x}_{c}} & -\frac{f}{\tilde{x}_{c}} & 0 \\
\frac{v}{\tilde{x}_{c}} & 0 & -\frac{f}{\tilde{x}_{c}}
\end{array}\right] \tilde{\mathbf{V}}_{B / C}\right)= \\
& =\mathbf{M}_{\tilde{\omega}_{B / C}}\left(f, \boldsymbol{r}_{c}\right)\left(\left[\begin{array}{l}
\dot{u} \\
\dot{v}
\end{array}\right]-\mathbf{M}_{\bar{v}_{B / C}}\left(f, \boldsymbol{r}_{c}\right) \tilde{\mathbf{V}}_{B / C}\right)
\end{aligned}
$$

Finally, the estimated angular velocity $\tilde{\boldsymbol{\omega}}_{k}$ to be used in Eq.

Error! Reference source not found. is obtained by rotating $\tilde{\boldsymbol{\omega}}_{k}=\mathbf{R}\left(\tilde{\mathbf{q}}_{k}\right) \tilde{\boldsymbol{\omega}}_{B / C}$ from the camera frame to the asteroid frame. Including other points' measurements, to give additional information and filter the error, $\boldsymbol{\omega}_{B / C}$ can be estimated using the batch least square

$$
\boldsymbol{\omega}_{B / C}=\left[\begin{array}{c}
\mathbf{M}_{\omega_{B / C}}\left(f, \mathbf{r}_{c, 1}, \mathbf{p}_{c, 1}^{a}\right) \\
\vdots \\
\mathbf{M}_{\omega_{B / C}}\left(f, \mathbf{r}_{c, N}, \mathbf{p}_{c, N}^{a}\right)
\end{array}\right]^{0}\left[\begin{array}{c}
\dot{u}_{1} \\
\dot{v}_{1} \\
\dot{u}_{N} \\
\dot{v}_{N}
\end{array}\right]-\left[\begin{array}{c}
\mathbf{M}_{\mathbf{v}_{B / C}}\left(f, \mathbf{r}_{c, 1}, \mathbf{p}_{c, 1}^{a}\right) \\
\vdots \\
\mathbf{M}_{\mathbf{v}_{B / C}}\left(f, \mathbf{r}_{c, N}, \mathbf{p}_{c, N}^{a}\right)
\end{array}\right]^{+} \mathbf{v}_{B / C}
$$

where the $\diamond$ sign stands for pseudo-inverse, and all the points give equal contribution to the solution (i.e. the weight associated to their corresponding information is equal to 1 ). The algorithm allows extracting velocity and attitude rates from at least three tracked feature points from two consecutive frames.

As an example, Figure 3 reports the error on the estimation of the angular velocity during a 14 days operations time.

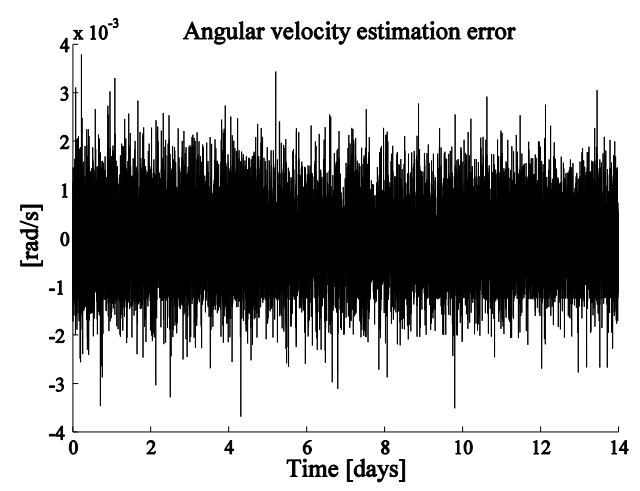

Figure 3: Angular velocity estimation error.

A total of 10 features were considered at each time. The pixelization error $S_{p}$ is equal to the dimension of the pixel which was set to $75 \mu \mathrm{m}$ (equivalent to the one of a camera with 2000 pixels and a screen width of $15 \mathrm{~cm}$ ). The asteroid surface is known with an accuracy $\boldsymbol{\zeta}_{\text {surface }}$ of $15 \mathrm{~cm} \mathrm{(3- \sigma )}$

and the bias $\mathbf{x}_{\text {CoM }}^{\text {bias }}$ on the position of the barycentre is $20 \mathrm{~cm}$ $(3-\sigma)$. The position and velocity of the spacecraft, $\delta \tilde{\mathbf{r}}^{h}$ and $\delta \tilde{\mathbf{r}}^{h}$, are assumed to be estimated with an accuracy of 20 $\mathrm{cm}$ and $0.1 \mathrm{~mm} / \mathrm{s} \quad(3-\sigma)$ respectively. An attitude determination error on 2 axes of $10^{-3}$ degrees is also considered. The figure shows that with the assumed measurement errors, the system is able to determine an angular rate as precise as few milliradians per second.

\section{Results}

We present here the preliminary analysis. We considered a cylindrical and a parallelepiped shape with different masses for our analysis.

First we have to underline an important consequence of our method choice for the case of a cylindrical satellite. In the case the geometrical axes are identical to the inertial one, a control torque cannot be applied along the height of the cylinder, since the normals to its surface are aligned with the control arm (producing a zero control torque along the longitudinal direction). In the case the geometrical and principal axes of inertia are distinct, a full matrix of inertia will favour the transfer of momentum from the non controllable axis to the controllable ones.

For the first set of analyses, we considered a spacecraft with a size and mass comparable to Envisat's bus in the first scale. Table 2 reports the characteristics we used. The mass is the same as the one of Envisat at the end of its life.

Table 2. Envisat like satellite characteristics ( $a-b$ transversal dimensions, $h$ height longitudinal; x-y transversal, z longitudinal axes) and initial angular velocity.

Total mass

Size

$h=9 \mathrm{~m}$

MOI

$\omega_{\mathrm{x}}=0.962 \mathrm{deg} / \mathrm{s}$

The dimensions are slightly smaller than the actual dimensions of Envisat (see [9]), which is a conservative choice because the resulting control torque is lower than the one achievable with bigger control arms. Nonetheless we used the same value for the matrix of inertia (MOI) as in [10].

We considered a random initial velocity of magnitude 3.5 $\mathrm{deg} / \mathrm{s}$ resulting from the recent observations as in [10]. All along the analysis the initial quaternion vecor was assumed as: 
$q_{0}=[0.2378,0.5420,0.5305,0.6067]^{T}$

Figure 4 shows the trend for the angular velocity components and magnitude during 14 days of control operations for a cylinder (where the radius is $2 \mathrm{~m}$ equal to $\mathrm{b} / 2$ or $\mathrm{c} / 2$ ). During this period the rotation rate is reduced to $1 \mathrm{deg} / \mathrm{s}$, less than one third of the initial value.

The angular velocity decreases slowly after the components on the transversal directions reach a nearly null velocity. Nonetheless if one continues operating the laser for a longer period, the spacecraft rotation will halt completely.

Moreover if the laser was allowed to hit flat surfaces, such as solar panels, parallel to the longitudinal axis, it would be possible to exert a direct control torque along this direction and reduce the rotation more rapidly.

This is the case of the parallelepiped satellite as shown in Figure 5. On the contrary of the previous case, the angular velocity descreases quite steeply. The rotational motion is almost halted completely in less than 1.5 days. The control
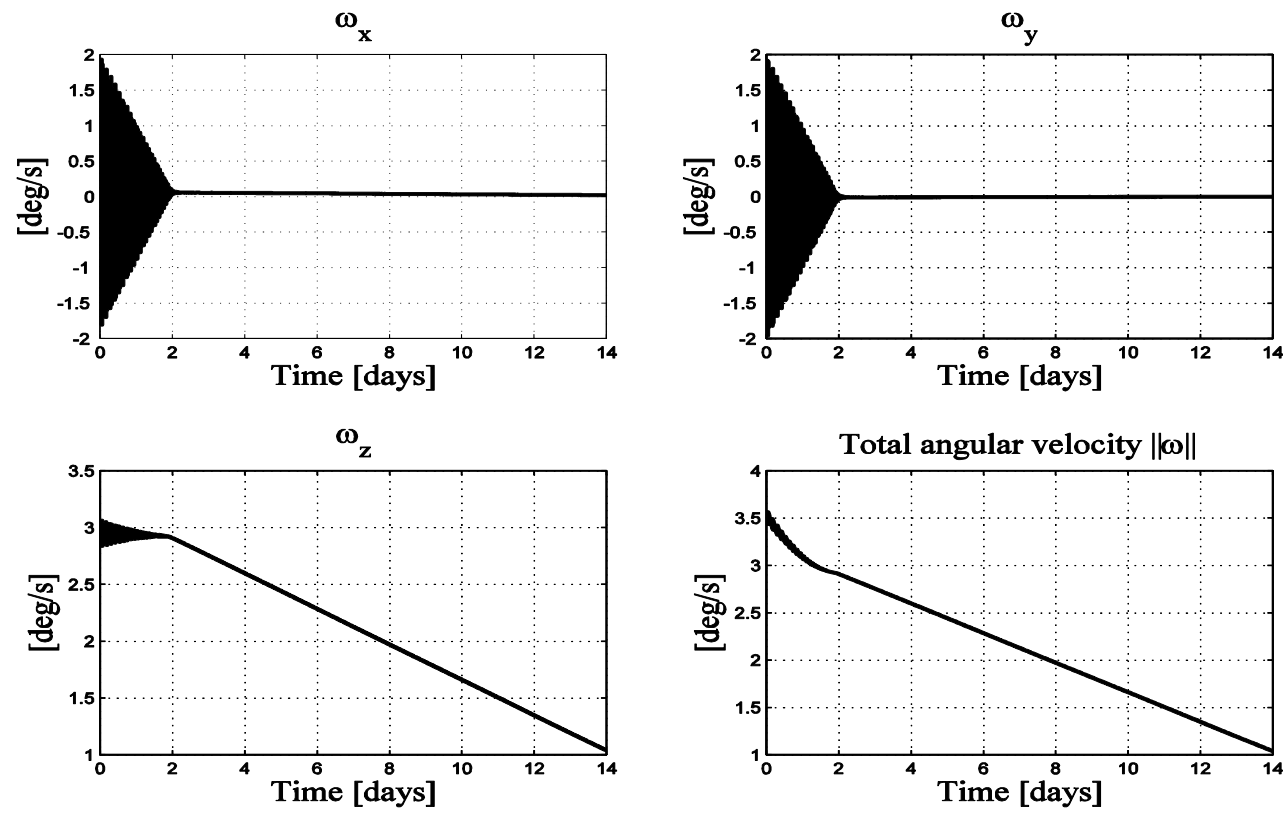

Figure 4. Envisat like satellite - cylindrical shape - angular velocity trend.
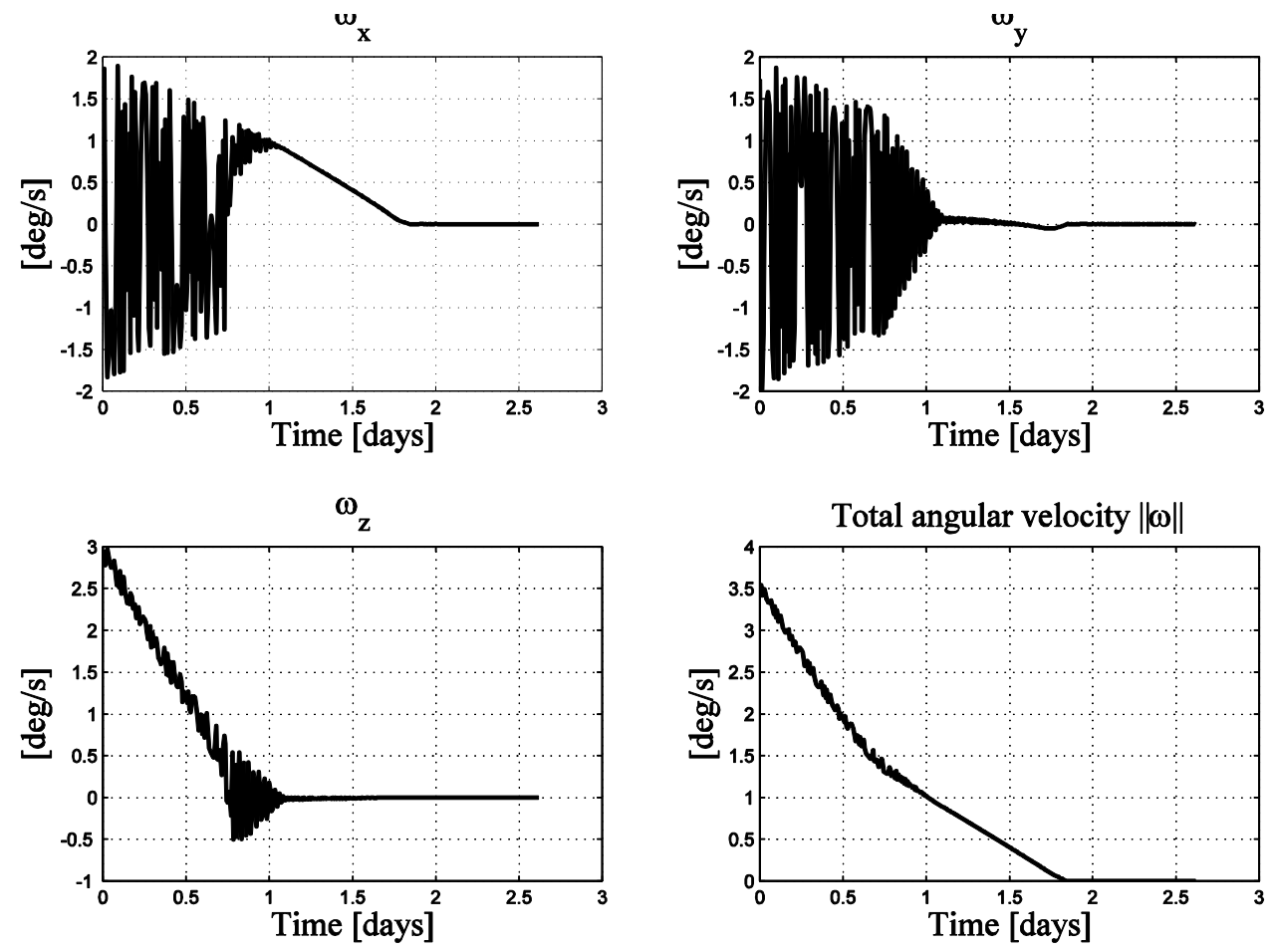

Figure 5. Envisat like satellite - parallelepiped shape - angular velocity trend. 
torque tends to always counteract the highest controllable component of the angular velocity at any time. The result here shows the third component decreasing concurrently with the other ones whereas in the cylindrical case this. As pointed out above, in this case a direct control of all the components is possible, while in the previous case the control has to rely on the momentum transfer between components whose effectiveness is limited by the magnitude of the cross elements of the matrix of inertia.

In the second case we analysed a 1 ton spacecraft with characteristics reported in Table 3 . Regarding the matrix of inertia we simply scale the Envisat one down proportionally to the current mass. The initial angular velocity is the same as the previous case (see Table 2).

Table 3. Small satellite characteristics ( $a-b$ transversal dimensions, $h$ height longitudinal; $\mathrm{x}-\mathrm{y}$ transversal, $\mathrm{z}$ longitudinal axes).

Total mass $1000 \mathrm{~kg}$

Size $h=2 \mathrm{~m}$ $a=1 \mathrm{~m}$ $b=1 \mathrm{~m}$

MOI

$\begin{array}{ll}I_{x x} & 9775.5 \mathrm{~kg} \mathrm{~m}^{2} \\ I_{y y} & 9450.9 \mathrm{~kg} \mathrm{~m}^{2} \\ I_{z z} & 1288.9 \mathrm{~kg} \mathrm{~m}^{2} \\ I_{x y} & 26.1 \mathrm{~kg} \mathrm{~m}^{2} \\ I_{x z} & -164.4 \mathrm{~kg} \mathrm{~m}^{2} \\ I_{z y} & 30.1 \mathrm{~kg} \mathrm{~m}^{2}\end{array}$

Figure 6 show the trend in the case of cylindrical shape. With respect the Envisat like satellite, the lower MOI allows the angular velocity go down to $0.4 \mathrm{deg} / \mathrm{s}$ during the same interval of time.

In the same way, also the parallelepiped case shows faster convergence with the angular velocity going to zero in less than half a day, see Figure 7.

\section{Conclusions}

This paper presented a method to control and decrease the angular velocity of a debris using laser ablation technique. The method we used considered to point the laser beam on the surface of the object such that the resulting control torque acts in the opposite direction of the instantaneous angular rate. Given that the ablative force is directed as the local normal to the surface, the control torque could not be directed exactly in the opposite direction. For this reason we proposed to point the laser such as to minimize the undesired control torque, which implies to minimize the angle between the direction of angular velocity and the opposite of the control torque. The designed control torque required determining the instantenous angular velocity. For this reason we estimated this quantity using the optical flow method.

We tested different inertia and different shapes of tumbling debris basing the simulation on actual flight data retrieved from the Envisat-4 satellite. As expected the higher is the mass, i.e. the inertia of the object, the longer is the time the control needs to be applied. Nonetheless we saw that the geometry plays a decisive role with this method. Using a cylinidrical shape, it would have not been possible to fully control the debris. This was achieved only because of the presence of extra diagonal elements in the matrix of inertia
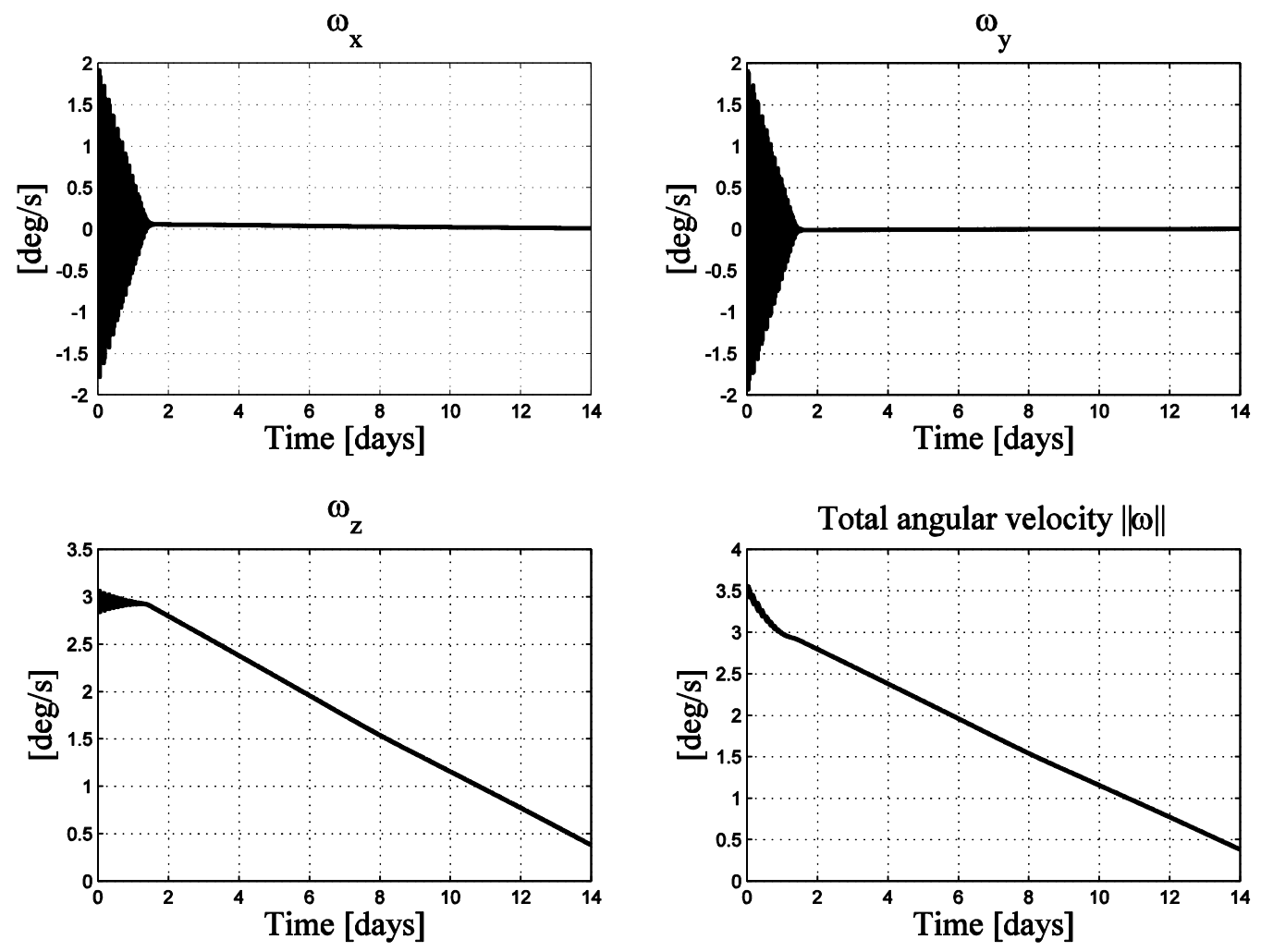

Figure 6. Small satellite - cylindrical shape - angular velocity trend. 

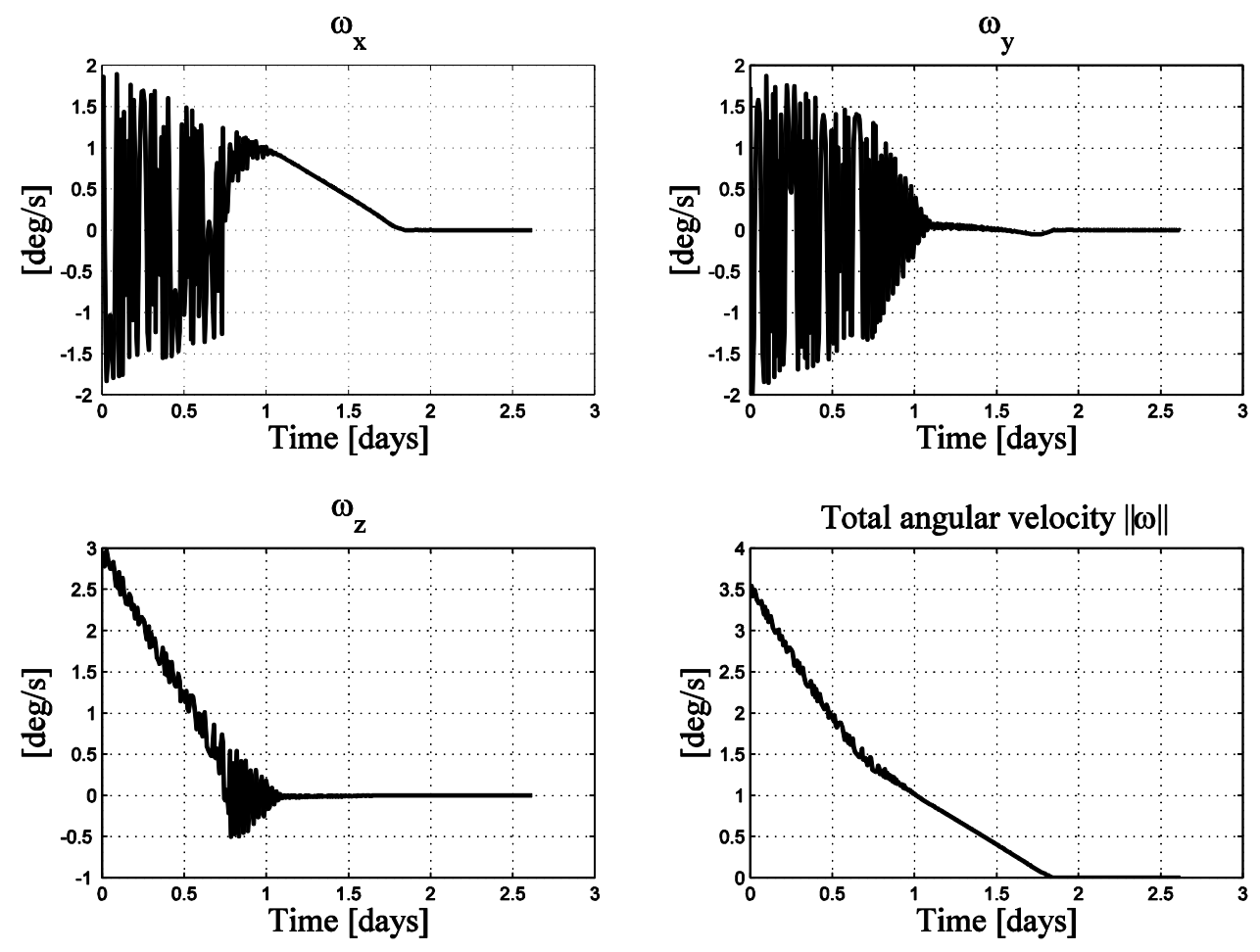

Figure 7. Small satellite, parallelepiped shape - angular velocity trend.

axis. Anyway detumbling a cylindrical debris required about one order of time more than detumbling a parallelepipedal debris with the same inertia and mass. The presence of very small cross inertial terms would affect the transfer of momentum between the components, increasing the duration of laser operations. Fortunately most of the debris present appandages or solar arrays which could be exploited for this purpose. This fact leads to a couple of consideration that will be necessary to model to obtain precise information about the effectiveness of this method.

We need to have a precise representation of the shape of the debris as well as the corresponding composition, because the bus composition is different from the solar arrays one, for instance. Then we must take into account the effect of the laser ablation on the mass and inertia of the debris. A more complicate issue is represented by the fact that the laser could perforate thin panels of the debris, making it impossible to actually control its rotation.

In this paper we assumed that the control torque is predominant in the rotational dynamics, but this will not be the case considering longer operation, smaller control torque and large solar arrays. For this reason next work will consider the effects of the gravity gradients, as well as solar radiation pressure and magnetic field. Finally we will implement also the laser carrying spacecraft dynamics to model the effects of the close range control.

\section{References}

[1] Caubet, A., Biggs, J., 2014. Design of an attitude stabilization electromagnetic module for detumbling
Aerospace and Electronic Systems, 01.03.2014, p. 113.

[2] Vetrisano, M., Colombo C., Vasile M., 2013. Asteroid Rotation and Orbit Control via Laser Ablation. Submitted to the Journal of Advances in Space Research

[3] Vasile M., Colombo C.: 2008, Optimal Impact Strategies for Asteroid Deflection. Journal of Guidance, Control, and Dynamics, Vol. 31, No. 4, July-August 2008, pp. 858-872, doi: 10.2514/1.33432.

[4] Vasile M., Vetrisano M., Gibbings A., Garcia Yarnoz D., Sanchez Cuartielles J-P., Burns D., Hopkins J-M., Colombo C., Branco J., Wayman A., Eckersley S.: 2013, 'Light Touch2, Effective Solutions to Asteroid Manipulation', ESA Final report, SysNova Study, ESA Reference Nos.: 12/X02, 12/X03, February 2013.

[5] Vasile M., Vetrisano M., Gibbings A., Garcia Yarnoz D., Sanchez Cuartielles J-P., Hopkins J-M., Burns D., McInnes C., Colombo C., Branco C., Wayman A., Eckersley S.: 2013b, 'Light-Touch2:2013, A LaserBased Solution for the Deflection, Manipulation and Exploitation of Small Asteroids', Planetary Defense Conference 2013, Flagstaff, USA, IAA-PDC13-04-22.

[6] Kahle, R., Kuhrt, E., Hahn, G., Knollenberg, J.: 2006, Physical limits of solar collectors in deflecting Earththreatening asteroids, Aerospace Science and Technology Vol. 10, pp. 253-263.

[7] Siegman, A. E.: 1986, Lasers. University Science Books. pp. 664-669. ISBN 0-935702-11-3.

[8] Longuet-Higgins, H.C. and Prazdny, K.: 1980, The 
interpretation of a moving retinal image. Proceedings of the Royal Society of London B 208, 385-397.

[9] https://earth.esa.int/web/guest/missions/esaoperational-eo-missions/envisat/satellite/spacesegment

[10] Bastida Virgili, B., Lemmens, S., Krag, H., 2014. Investigation on Envisat attitude motion. ESA/ESOC Space Debris Office e.Deorbit Workshop, 06/05/2014.

[11] Vasile M., Maddock C.: 2012, Design of a Formation of Solar Pumped Lasers for Asteroid Deflection, Advances in Space Research, 2012.

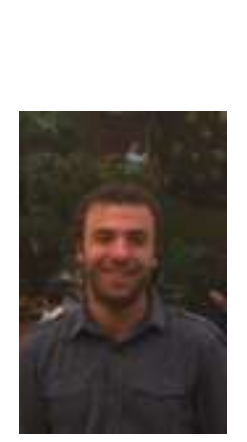

\section{Biography}

Massimo Vetrisano earned his BSC and MSc in Aerospace Engineering at Politecnico di Milano, Milan. He is finalizing his PhD under the guidance of Professor M. Vasile from the University of Strathclyde, Glasgow, where Massimo carried out his doctorate His research was centred on autonomous guidance, navigation and control systems for missions to minor celestial bodies. In particular, he focussed on strategies and algorithms to increase reliability and minimise the impact of failure. Massimo took part to European Student Moon Orbiter (ESMO) as flight dynamics specialist. He was also been involved as researcher in studies for end-of-life and disposal concepts and asteroid deflection missions. Thanks to the latter work, in 2013 he won the Space Generation OHB Move an Asteroid Competition in Beijing. Finally Massimo worked at Compagnia Generale per lo Spazio S.p.a., an OHB company, for about 1 year before starting hi PhD. His research interests include: Optimal estimation and control, uncertainty quantification, space robotics and autonomous navigation.

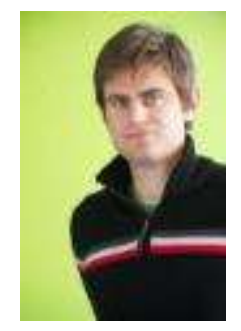

Nicolas Thiry is an Early Stage Researcher within Stardust Marie Curie ITN at the University of Strathclyde, Glasgow. His current work involves the study and comparison of different approaches for the active removal/deflection of uncooperative targets. Before this, Nicolas got a first master's degree in mechanical Engineering from Universite Catholique de Louvain and a second master's degree in fluid dynamics at the von Karman Institute where his research on supersonic inlets was awarded the Belgian government prize. His professional experience includes one year as a young graduate trainee in the structure section of the European Space Research and Technology Centre (ESA/ESTEC) in the Netherlands and one year as a vibro/aero-acoustic engineer for Free Field Technologies, MSC Software company.

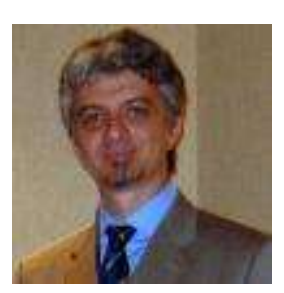

Massimiliano Vasile is currently Professor of Space Systems Engineering in the Department of Mechanical \& Aerospace Engineering at the University of Strathclyde. Formerly in 2004, he was the first member of the ESA Advanced Concepts Team and initiator of the ACT research stream on global trajectory optimisation, mission analysis and biomimicry. His research interests include Computational Optimization, Robust Design and Optimization Under Uncertainty exploring the limits of computer science at solving highly complex problems in science and engineering.

He developed Direct Transcription by Finite Elements on Spectral Basis for optimal control, implemented in the ESA software DITAN for low-thrust trajectory design. He has worked on the global optimisation of space trajectories developing innovative single and multi-objective optimisation algorithms, and on the combination of optimisation and imprecise probabilities to mitigate the effect of uncertainty in decision making and autonomous planning. More recently he has undertaken extensive research on the development of effective techniques for asteroid deflection and manipulation. Prof Vasile is currently leading Stardust, an EU-funded international research and training network on active debris removal and asteroid manipulation. 
